\title{
Frozen spatial chaos induced by boundaries
}

\author{
Víctor M. Eguíluz, ${ }^{*}$ Emilio Hernández-García, Oreste Piro, and Salvador Balle \\ Instituto Mediterráneo de Estudios Avanzados IMEDEA ${ }^{\dagger}$ (CSIC-UIB) E-07071 Palma de Mallorca, Spain
}

(Received 4 June 1999)

\begin{abstract}
We show that rather simple but nontrivial boundary conditions could induce the appearance of spatial chaos (that is stationary, stable, but spatially disordered configurations) in extended dynamical systems with very simple dynamics. We exemplify the phenomenon with a nonlinear reaction-diffusion equation in a twodimensional undulated domain. Concepts from the theory of dynamical systems, and a transverse-single-mode approximation are used to describe the spatially chaotic structures. [S1063-651X(99)01812-7]
\end{abstract}

PACS number(s): 05.45.-a

\section{INTRODUCTION}

In the past few decades, considerable understanding of the phenomenon of temporal chaos in dynamical systems of few degrees of freedom has been achieved [1-3]. On the other hand, spatiotemporal chaos in extended dynamical systems with infinitely many degrees of freedom is currently under very active investigation $[4,5]$. It is remarkable however, that an area of problems laying somehow between the two extremes has not received so much attention, namely, purely spatial chaos as a stationary attractor of extended dynamical systems [6-11].

The possible existence of this kind of attractors was suggested by Ruelle [12] in the context of equilibrium phases. He pointed out the parallelism between a time-dependent differentiable dynamical system and the space dependence of equilibrium states in statistical mechanics. He then raised the question as to whether the existence of turbulent crystals could be the natural next step towards complexity after spatially homogeneous, periodic, and quasiperiodic equilibrium phases have been found. Newell and Pomeau [13] gave some conditions under which such a turbulent crystal would exist in pattern-forming systems described by a free energy. Theoretical and experimental work on modulated phases and commensurate-incommensurate transitions $[14,15]$ represent additional concrete results along these lines.

In the context of fluid dynamics, the existence of spatially chaotic, but temporally steady solutions would also fill a conceptual gap between two well-studied complex phenomena: Lagrangian chaos, and Eulerian chaos or turbulence. The former refers to the chaotic motion of a fluid parcel which might occur even in laminar and, in three dimensions, steady flows [16-18]. On the other extreme, the road to turbulence is usually associated with a hierarchy of instabilities leading to increasingly spatiotemporally chaotic Eulerian velocity fields. Frozen spatial chaos would then refer in this context to a third possibility: a stationary flow spatially chaotic in the Euler description.

By now, many extended dynamical systems displaying spatial chaos have been identified. Most of the studies are concerned with one dimensionally extended systems. They are especially suitable to analysis because their steady-state

\footnotetext{
*Electronic address: victor@imedea.uib.es

†URL: http://www.imedea.uib.es/Nonlinear
}

configurations depend just on the unique spatial coordinate. These configurations are solutions of sets of ordinary differential equations (the spatial dynamical system) with the space variable as the independent variable. The standard theory of low-dimensional dynamical systems can be used to describe such configurations, by just considering the spatial coordinate as a fictitious time. Rigidly traveling waves with spatial chaotic structure can also be considered as a case of spatial chaos in a moving reference frame [8-10].

Spatial chaos may appear when the spatial dynamical system has a sufficiently high dimensional phase space. This high dimensionality may arise from either (a) the presence of high-order spatial derivatives in a single evolution equation as in the cases of the Swift-Hohenberg equation [19,20], and Kuramoto-Sivashinsky and related models [9,11], (b) the coupling of several fields each one satisfying a lower order differential equation as in excitable media [10] and in (the real and imaginary parts of) the complex Ginzburg-Landau equation [8] which supports chaotic traveling waves, or (c) explicit space-dependent forcing terms as in [6] or [21].

Consideration of two-dimensional spatial chaos has been very scarce. In the absence of a simple connection with conventional dynamical systems theory, the very concept of chaos in the spatial configuration should be properly defined for the general two-dimensional case. A rather complete formalism generalizing dynamical system tools (entropies, dimensions,...) to multidimensional spatial chaos has been developed [7,22], and some examples examined [7,20]. On the other hand, as the number of dimensions increases, a much larger variety of nontrivial boundary condition classes surely leads to a greater richness in the expected properties of the steady field configurations. A well-posed question is then whether relatively simple boundary conditions may lead to steady spatially chaotic configurations. The main purpose of this paper is to address this question.

In addition to the existence of chaotic spatial configurations, it is important also to study their stability in time. A stationary state will only be physically observable if it is stable or at least long-lived. It turns out that the temporal stability of the stationary solutions is in general unrelated to the stability of these configurations considered as orbits of the spatial dynamical system. In the examples cited above, there are cases of both stable and unstable space-chaotic configurations, but instability seems to be more frequent. As a 
consequence, spatial chaos has been generally considered of limited physical relevance.

In this paper we show that rather simple undulated striplike domain shapes can induce, in a very simple nonlinear extended dynamical system, the formation of patterns that are both spatially chaotic and temporally attracting. The kind of modulated boundaries we use could be easily implemented in standard experimental pattern-formation setup's such as Faraday waves, convection cells, or open flows. In fact, our work was originally motivated by the observation, in a fluid dynamics experimental setup consisting of a periodic array of pipe bends, that the transverse profile of the steady flow does not necessarily repeat itself with the same periodicity of the array [17].

In Sec. II we present the particular model that we study and perform a preliminary analysis of its behavior. In Sec. III a single-transverse-mode approximation is introduced and we use it to predict the existence of boundary-induced spatial chaos. Numerical simulations are presented in Sec. IV to substantiate our claims beyond the validity of the previous approximation. Finally, we summarize the results and open problems in the Conclusions.

\section{A REACTION-DIFFUSION EQUATION IN A STRIP-SHAPED DOMAIN}

As stated before, the application of the theory of dynamical systems to the study of stationary spatial configurations of one-dimensionally extended systems is direct. A stationary pattern satisfies, in general, a system of ordinary differential equations with the spatial coordinate as its independent variable which we can think of as a time. Parity symmetry in a spatial coordinate will appear as the time-reversal symmetry after reinterpretation of this coordinate as time.

The general study of spatial chaos in several spatial dimensions requires the notion of translational dynamical systems with $d$ times $[7,22]$. There are situations, however, where such formalism is not necessary because the two independent spatial directions are distinguished by the geometry of the system, so that one of them naturally plays the role of time. In this way, the spatial variation in one direction would be interpreted as time evolution of a one-dimensional field that only depends on the remaining spatial coordinate. Particularly suited to our approach will be the case of twodimensional extended systems in strip-shaped regions much longer (ideally infinite) in the timelike direction than in the spacelike one. If the strip is narrow enough, only patterns composed of one or few transverse spatial modes will be allowed and spatial chaos could be readily defined and identified in terms of the usual concepts of dynamical systems theory.

In order to concentrate on spatial chaos purely induced by boundary effects, we consider a very simple model equation containing only up to second-order derivatives and a single field variable, a reaction-diffusion equation of the FisherKolmogorov type:

$$
\partial_{t} \psi=\nabla^{2} \psi+a \psi-\psi^{3},
$$

with appropriate boundary conditions for the real field $\psi(\mathbf{x}, t)$. The real linear coefficient $a$ can be absorbed rescaling the variables, but we find convenient to keep it explicit in the equation. Equation (2.1) appears in several contexts including phase transitions, where it takes the name of real Ginzburg-Landau equation or time-dependent GinzburgLandau model [23], and population dynamics [24]. The dynamics of Eq. (2.1) can be written as purely relaxational [25] in a functional Lyapunov potential $V[\psi]$ :

$$
\partial_{t} \psi=-\frac{\delta V[\psi]}{\delta \psi}
$$

with

$$
V[\psi]=\int_{D} d \mathbf{x}\left(\frac{1}{2}|\nabla \psi|^{2}-\frac{a}{2} \psi^{2}+\frac{1}{4} \psi^{4}\right)+S[\psi],
$$

where the integral is over the domain $D$. The surface term $S[\psi]$ takes into account the effects of boundary conditions over the domain limit, and it vanishes when periodic, null Dirichlet $(\psi=0)$ or null Neumann $\left(\partial_{n} \psi=0\right)$ boundary conditions are specified. It follows from Eq. (2.2) that $V$ can only decrease with time. The relaxational character of Eq. (2.2) implies also that the only asymptotic states are fixed points. Therefore, this model does not display any limitcycle oscillations or more complex dynamics such as temporal chaos in any number of spatial dimensions.

Equation (2.1) has been extensively studied in one and two dimensions. In one dimension, for infinite systems, we have the following situations: For $a<0, \psi=0$ is the only stationary solution and is stable under time evolution. At $a$ $=0$ a pitchfork bifurcation occurs and the former trivial solution loses its stability. For $a>0$ some of the stationary solutions are the following:

(1) Homogeneous solutions: $\psi(x, t)=0$, and $\psi(x, t)=$ $\pm \sqrt{a} \equiv \psi_{ \pm}$.

(2) Kink type solutions: $\psi(x, t)= \pm \sqrt{a} \tanh (\sqrt{a / 2} x)$.

(3) Periodic solutions: $\psi(x, t)=\sqrt{a-k^{2}} \sin (k x)+\ldots$, where the ellipsis stands for higher-order harmonics.

Only $\psi_{ \pm}$and the kinks are now linearly stable.

For finite systems, the boundary conditions normally restrict the variety of solutions either by selecting some values of $k$, or by favoring either the kink or the $\psi_{ \pm}$solutions. Additionally, the boundary conditions may have as an effect a shift in the pitchfork bifurcation point $a=0$ to a different value $a_{c} \neq 0$ and a change in the coefficients appearing in the solutions.

The typical time evolution of initial field distributions leads to the formation of domains where values of $\psi$ close to either $\psi_{+}$or $\psi_{-}$dominate. These domains are separated by kink- or anti-kink-type walls that can move into each other producing a mutual annihilation. By this mechanism, small domains disappear and feed the larger domains whose sizes then increase logarithmically in time until one of the stationary solutions, prevailing by chance, takes over the whole system.

In two dimensions, the dynamics typically consists of the coarsening of domains of $\psi_{+}$and $\psi_{-}$phases whose typical size grows as the square root of time [23]. Interfaces between the two phases are locally similar to the one-dimensional kink solutions. The gradient term in Eq. (2.3) is important at the interfaces giving a positive contribution to the Lyapunov potential. Since the dynamics always minimizes $V$, it tends to 


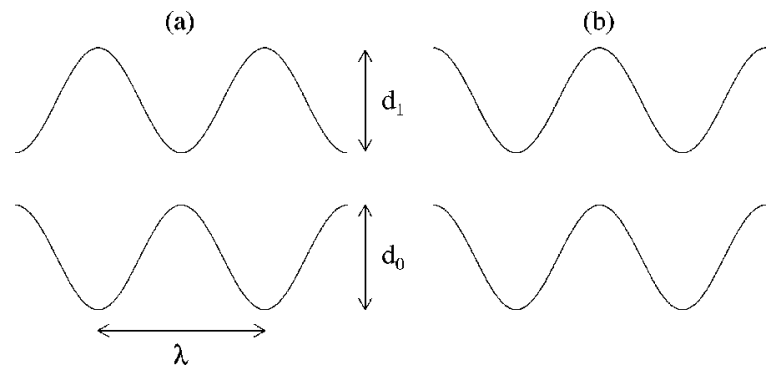

FIG. 1. Examples of stripped channels enclosed in oscillating walls: $\alpha=2 \pi / \lambda ; d_{0}=d_{1}$, and (a) $\phi=\pi$, (b) $\phi=0$.

reduce the length of these interfaces. This reduction is achieved by the shrinking and ulterior collapse of the smallest domains to contribute to the (square-root) growth of the remaining ones.

It was shown by Collet [26] in a more general context that the time evolution and final states of Eq. (2.1) in finite domains are similar to those in an infinite system except in a boundary layer around the border whose size depends on the $a$ parameter. This result holds in both one and two dimensions. Thus, in order to observe the influence of boundaries on pattern evolution, we need to consider a domain small enough at least in one of the directions. In a stripped domain, elongated in the $x$ direction, this small dimension will be the transverse $y$ direction. The domain will be limited in this transverse dimension by the boundaries $y_{0}(x)$ and $y_{1}(x)$, where the function $\psi(x, y, t)$ will take values $\psi_{0}(x) y \psi_{1}(x)$, respectively (Dirichlet conditions).

In the one-dimensional case, conventional dynamical systems theory implies that there are no chaotic stationary solutions to Eq. (2.1) because the spatial dynamical system is just a second-order ordinary differential equation. However, chaos can arise if some $x$-dependent periodic forcing is added to the equation. These arguments do not apply directly to the two-dimensional case. However, it is tempting to think of undulations of the lateral boundaries as a kind of periodic forcing on the longitudinal coordinate. This suggests the possibility of finding chaotic structures in the $x$ direction induced by undulated boundaries.

As a particular case we consider domains limited by two sinusoidal boundaries. With applications to hydrodynamics in mind we think of these domains as channels with sinusoidal banks.

$$
\begin{gathered}
y_{1}(x)=\frac{d_{1}}{2}[1-\cos (\alpha x)], \\
y_{0}(x)=-1-\frac{d_{0}}{2}[1+\cos (\alpha x+\phi)] .
\end{gathered}
$$

Here $d_{1}, d_{0}$ are the amplitude of the undulation of each bank, $\alpha$ is the spatial frequency which we assume to be the same for both banks and $\phi$ is their mutual phase mismatch. Figure 1 shows a few typical shapes for our channel-like domains. The case $d_{1}=d_{0}$ and $\phi=\pi$ gives a sausage-shaped channel with symmetrically and sinusoidally varying width. On the other hand, $d_{1}=d_{0}$ and $\phi=0$ sets the boundaries in phase and corresponds to a domain with the form of a sinusoidally meandering channel of constant $y$ width.
We stress that we want to consider the simplest situation that may display spatial chaos. Consideration of more complex equations exhibiting spatial chaos even with simple boundaries, or more complex boundaries such as incommensurate oscillations for the upper and lower banks (corresponding to quasiperiodic forcing) would only enrich the complexity of stationary solutions.

A complete definition of the model requires also the specification of boundary conditions on the longitudinal $x$ direction. The analogy with a temporal variable would be better for domains infinite in the $x$ direction, with only the weak requirement of boundedness for $\psi$. However, an infinite domain is inadequate for the numerical approaches to be described below. In our calculations we would need to impose periodic boundary conditions (of period $L$ ) along the $x$ direction. In this way we are restricting the class of solutions to periodic orbits of period $L$ or less in the time-like coordinate. We will still be able to identify as spatially chaotic the configurations that have the maximal period $L$, provided this period increases and the periodic orbit approaches a chaotic trajectory as system size $L$ increases. Subtle considerations such as Lyapunov number computations for such limiting orbits will be addressed elsewhere.

To perform numerical simulations we can choose between several strategies. If we are only interested in stationary states, we can numerically solve the time-independent version of Eq. (2.1) by means of finite elements or finite differences. These methods can be implemented to find solutions that may or may not be stable under time evolution. Another possibility is to follow the dynamics of the full Eq. (2.1) until a stationary state is reached. In this way, only attracting (i.e., stable) stationary solutions can be found (remind that only stationary attractors are allowed by this purely relaxational dynamics). In any case, a convenient way to handle the boundary conditions is to map the region limited by $y_{0}(x)$ and $y_{1}(x)$ (and by $x=0, L$ ) to a rectangular one: $\tilde{y}_{1}=1, \tilde{y}_{0}$ $=0$, and $x=0, L$. For arbitrary functions $y_{0}(x)$ and $y_{1}(x)$, the map $(x, y) \mapsto(x, \tilde{y})$, with

$$
\tilde{y}=\left(y-y_{0}\right) /\left(y_{1}-y_{0}\right),
$$

transforms Eq. (2.1) into an equation for $\widetilde{\psi}(x, \tilde{y}, t)$ $\equiv \psi(x, y, t)$ :

$$
\partial_{t} \widetilde{\psi}=\partial_{x x}^{2} \widetilde{\psi}+\mathcal{F}(x) \partial_{y y}^{2} \widetilde{\psi}+\mathcal{G}(x) \partial_{x \tilde{y}}^{2} \widetilde{\psi}+\mathcal{H}(x) \partial_{\tilde{y}} \widetilde{\psi}+a \widetilde{\psi}-\widetilde{\psi}^{3},
$$

where

$$
\begin{gathered}
\mathcal{F}(x)=\frac{1+\left(\Delta_{x} \tilde{y}+y_{0 x}\right)^{2}}{\Delta^{2}}, \\
\mathcal{G}(x)=-2 \frac{\Delta_{x} \tilde{y}+y_{0 x}}{\Delta}, \\
\mathcal{H}(x)=\frac{\tilde{y}\left(2 \Delta_{x}^{2}-\Delta \Delta_{x x}\right)-\Delta y_{0 x x}+2 \Delta_{x} y_{0 x}}{\Delta^{2}}, \\
\Delta(x)=y_{1}(x)-y_{0}(x),
\end{gathered}
$$




$$
\begin{gathered}
\Delta_{x}(x)=\frac{d}{d x} \Delta(x), \\
\Delta_{x x}(x)=\frac{d^{2}}{d x^{2}} \Delta(x), \\
y_{0,1 x}=\frac{d}{d x} y_{0,1} .
\end{gathered}
$$

Here $\Delta(x)$ is the transverse distance between the boundaries and gives the width of the strip. If this width does not vary along $x$, then $\Delta_{x}(x)=0=\Delta_{x x}(x)$.

The new transverse boundary conditions are

$$
\widetilde{\psi}(x, \tilde{y}=0)=\psi_{0}(x), \quad \widetilde{\psi}(x, \tilde{y}=1)=\psi_{1}(x) .
$$

A first observation is that the shape of the domain boundaries is reflected as a parametric forcing of the equation in the new coordinates. For example, for the simplest case of a meandering channel given by Eqs. (2.4) and (2.5) with $\phi$ $=0$, and $d_{0}=d_{1}=d$ [Fig. $\left.1(\mathrm{~b})\right]$ :

$$
\begin{gathered}
\partial_{t} \tilde{\psi}=\partial_{x x}^{2} \widetilde{\psi}+\mathcal{F}(x) \partial_{y \tilde{y}}^{2} \tilde{\psi}+\mathcal{G}(x) \partial_{x \tilde{y}}^{2} \tilde{\psi}+\mathcal{H}(x) \partial_{\tilde{y}} \tilde{\psi}+a \widetilde{\psi}-\widetilde{\psi}^{3} \\
\mathcal{F}(x)=\frac{1+\left(\frac{d \alpha \sin (\alpha x)}{2}\right)^{2}}{(1+d)^{2}} \\
\mathcal{G}(x)=-\frac{d \alpha \sin (\alpha x)}{1+d} \\
\mathcal{H}(x)=-\frac{d \alpha^{2} \cos (\alpha x)}{2(1+d)}
\end{gathered}
$$

Setting the right-hand side of Eq. (2.7) to zero in order to seek for stationary solutions, and thinking of $x$ as the time, we can view Eq. (2.7) as a nonlinear evolution equation for a one-dimensional field with a "time"'-periodic parametric driving due to the boundaries. Present knowledge on spatiotemporal chaos and pattern formation can, in principle, be applied to analyze the behavior of this resulting evolution equation. General results are not abundant, however. In Sec. III further approximations will be introduced in order to facilitate the analysis and establish the existence of stationary spatially chaotic solutions.

\section{SINGLE-TRANSVERSE-MODE APPROXIMATION}

For definiteness, in the rest of the paper we will consider just null Dirichlet boundary conditions, that is the field $\psi$ takes the value zero at the transverse boundaries: $\psi_{0}(x)$ $=\psi_{1}(x)=0$. In this case our model in the form of Eq. (2.7) has the trivial solution $\widetilde{\psi}(x, \tilde{y}, t)=0$. The stability analysis of this solution for the case of a rectangular domain of width $l$ leads to an eigenvalue problem for the linearized equation. For $a>a_{c}=(\pi / l)^{2}$ the eigenfunctions factorize into longitudinal $\left[\exp \left(i k_{x} x\right)\right]$ and transverse $\left[\sin \left(k_{y} \tilde{y}\right)\right]$ modes: $\widetilde{\psi}_{\lambda, \mathbf{k}}(x, \tilde{y}, t)=\exp (\lambda t) \exp \left(i k_{x} x\right) \sin \left(k_{\tilde{y}} \tilde{y}\right)$, with $k_{y}^{\sim}=(\pi / l) m, m$
$=1,2, \ldots, k_{x}$ real, $\lambda$ satisfying the dispersion relation $\lambda$ $=a-k^{2}$, and $k^{2}=k_{x}^{2}+k_{\tilde{y}}^{2}$. The unstable modes are then those satisfying the condition $k^{2}<a$. The first unstable mode corresponds to $\left(k_{x}, k_{\tilde{y}}\right)=(0, \pi / l)$, which becomes unstable at the critical value $a_{c}=(\pi / l)^{2}$. The transverse modes are discretized in multiples of $\pi / l$ due to the boundary condition. If in addition, we require $L$ periodicity in the longitudinal coordinate $x, k_{x}$ will be also discrete, but provided that $L \gg l$ this discretization will be much finer than the transverse one. The value of the parameter $a$ controls how many modes are linearly unstable. If the transverse size $l$ is small enough for the control parameter to satisfy the condition $(\pi / l)^{2}<a$ $<(2 \pi / l)^{2}$, there would be just one linearly unstable transverse mode, with many associated longitudinal unstable modes. Close enough to the instability threshold, we can try an approximate solution of the form $\widetilde{\psi}(x, \tilde{y}, t)$ $=A(x, t) \sin (\pi \tilde{y})$ and write an evolution equation for the amplitude $A(x, t)$ of the first transverse mode.

We are interested, however, in a domain which is not a rectangular strip but an undulated channel. The coordinate change bringing our domain into a rectangular one renders the variables nonseparable and the linear problem is no longer solvable analytically. However, for small deviations from the uniform channel a perturbation scheme can be used. In the same vein as in the preceding paragraph we try, for our undulated domain, the ansatz $\widetilde{\psi}(x, \tilde{y}, t)=A(x, t) \sin (\pi \tilde{y})$ $+\mathcal{O}\left(d_{0}, d_{1}\right)$, assuming that $a$ is close to the threshold imposed by the small $l$, and that the size of the channel undulations is small. We call this approach a single-transversemode approximation (STMA).

Projecting Eq. (2.7) onto the single-transverse mode present in the ansatz and neglecting higher-order contributions we get the following evolution equation for the amplitude $A$ :

$$
\partial_{t} A=\partial_{x x}^{2} A+\beta(x) \partial_{x} A+\omega^{2}(x) A-\frac{3}{4} A^{3},
$$

where

$$
\beta(x)=\frac{\Delta_{x}}{\Delta}
$$

and

$$
\begin{aligned}
\omega^{2}(x)= & a+\frac{\Delta \Delta_{x x}-2 \Delta_{x}^{2}}{2 \Delta^{2}}-\left(\frac{\pi}{\Delta}\right)^{2}\left(1+y_{1 x} y_{o x}\right) \\
& -\left(2 \pi^{2}-3\right) \frac{\beta^{2}}{6} .
\end{aligned}
$$

We have checked that a more rigorous, but lengthy, approach based on a multiple scale expansion leads to the same result [27]. The stationary patterns satisfy the timeindependent version of Eq. (3.1). In terms of the coordinate $x$ considered as a time, one can view this spatial dynamical system as a parametrically forced nonlinear oscillator: domain undulations provide a periodic driving on the frequency of the system $\omega^{2}(x)$. In turn, $\beta(x)$ is a "dissipation" term which can be positive or negative depending on $x$. This $x$ 
modulation of $\beta$ comes from the longitudinal variation of the vertical width. The integral of the "dissipation" $\beta(x)$ on one period of the oscillating boundaries $T=2 \pi / \alpha$ is $\int_{x_{0}}^{x_{0}+T} \beta(x) d x=\ln \left[\Delta\left(x_{0}\right)\right] /\left[\Delta\left(x_{0}+T\right)\right]=0$. This shows that although system (3.1) is locally dissipative, it is effectively conservative over one period of the modulation. This implies, in particular, that the stroboscopic map associated with the system (3.1) is area preserving.

In the general case, Eq. (3.1) can be simplified by removing the dissipation term with the change: $A(x, t)$ $=\exp \left[-\frac{1}{2} \int \beta(x) d x\right] \rho(x, t)=\rho(x, t) / \sqrt{\Delta(x)}$. The new equation reads

$$
\begin{gathered}
\partial_{t} \rho=\partial_{x x}^{2} \rho+\Omega^{2}(x) \rho-\frac{3}{4 \Delta} \rho^{3}, \\
\Omega^{2}=a-\left(\frac{\pi}{\Delta}\right)^{2}\left(1+y_{1 x} y_{o x}\right)-\frac{\pi^{2}+3}{12} \beta^{2} .
\end{gathered}
$$

A particular case occurs when the transverse distance between the two channel borders does not vary along the longitudinal direction, so that the dissipation term vanishes identically, i.e., $\beta(x)=0$. For the sinusoidal channel this happens when $\phi=0$ and $d_{0}=d_{1}=d$ [Fig. 1(b)]. In this case, the amplitude equation is reduced to

$$
\partial_{t} A=\partial_{x x}^{2} A+\omega^{2}(x) A-\frac{3}{4} A^{3}
$$

with

$$
\omega^{2}=a-\left(\frac{\pi}{1+d}\right)^{2}-\frac{(\pi d \alpha)^{2}}{4(1+d)^{2}} \sin ^{2}(\alpha x) .
$$

When $\partial_{t} A=0$, Eq. (3.6) is a dissipative Mathieu equation modified by the addition of a cubic nonlinear term or, equivalently, a parametrically forced Duffing oscillator. This equation is known to have chaotic solutions [28].

The general time-independent case of Eq. (3.1) [or Eq. (3.4)] reads

$$
\partial_{x x} A=-\omega^{2}(x) A+\frac{3}{4} A^{3}-\beta(x) \partial_{x} A
$$

In the absence of undulations $\left(d_{0}=d_{1}=0\right)$ parametric forcing and dissipation vanish and the equation is both Hamiltonian and integrable. It has a region in phase space $\left(A, A_{x}\right)$ close to the origin where motion is bounded and regular. Beyond the separatrices of the two saddle points $\left(A, A_{x}\right)$ $= \pm 2 / \sqrt{3}\left(a-\pi^{2}, 0\right)$, trajectories escape to infinity. When undulations are introduced, separatrices of the saddle points deform and may cross. It is well known that for perturbed Hamiltonian systems, separatrix intersections indicate the onset of chaos. In our system, in addition to chaotic bounded trajectories, separatrix intersections lead also to fractalization of the phase-space boundary dividing bounded and unbounded trajectories. Melnikov theory provides us with the tools to determine analytically the necessary conditions for separatrix intersection and the occurrence of chaos. Follow- (a)

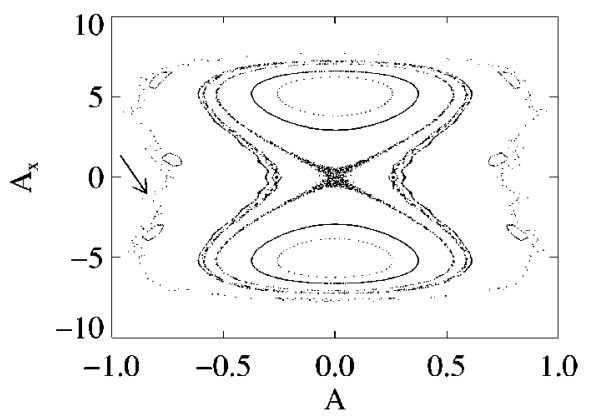

(b)

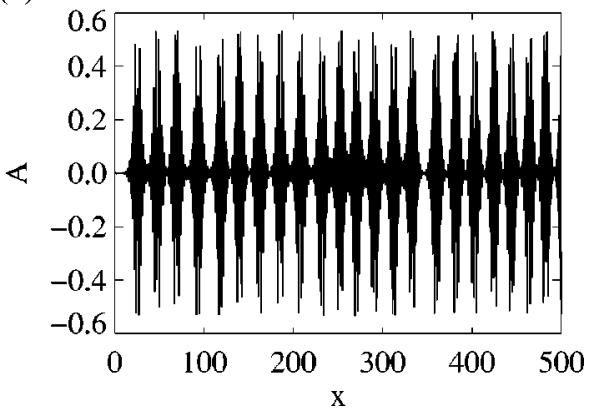

FIG. 2. (a) Stroboscopic Poincare map of the phase space of the system (3.8) for the values $d_{0}=d_{1}=1, \alpha=2 \pi, a=17, \phi=\pi$. KAM tori and chaotic trajectories in between are clearly seen. The arrow indicates the approximate location of the fractal boundary separating bounded and unbounded trajectories. (b) The chaotic configuration corresponding to the cloud of points surrounding the origin in the stroboscopic map.

ing [28], the Melnikov function $M(\theta)$ can be calculated for small $d$. For example, in the case $\phi=\pi, d_{0}=d_{1}=d$ one finds

$$
M(\theta)=f(a, \alpha) \sin (\alpha \theta) .
$$

The fact that the function $M(\theta)$ has zeros as a function of $\theta$ indicates that separatrix intersection and chaotic behavior occur. Chaotic behavior appears for arbitrarily small sizes of the channel undulations. We expect similar behavior to occur for other values of $\phi, d_{0}$, and $d_{1}$.

To illustrate the chaotic behavior of the stationary STMA (3.8) we show in Fig. 2(a) its numerical stroboscopic map. The dots are values of $\left(A, A_{x}\right)$ at multiples of the forcing period $T=2 \pi / \alpha$ for a set of initial conditions. Several regions dominated by chaotic trajectories and separated by KAM tori (the closed curves corresponding to quasiperiodic solutions) are clearly recognized in the picture. Also, the approximate location of the fractal boundary separating bounded trajectories from those escaping to infinity is pointed out by an arrow. Melnikov analysis also implies the existence of a dense set of unstable periodic orbits in the vicinity of the separatrix intersection on both sides of the fractal boundary mentioned before. These periodic orbits and the fact that they constitute a skeleton of the chaotic trajectories, are important for our analysis because the periodic boundary conditions in the $x$-direction select them out from the uncountable many other possible solutions of Eq. (3.8). 


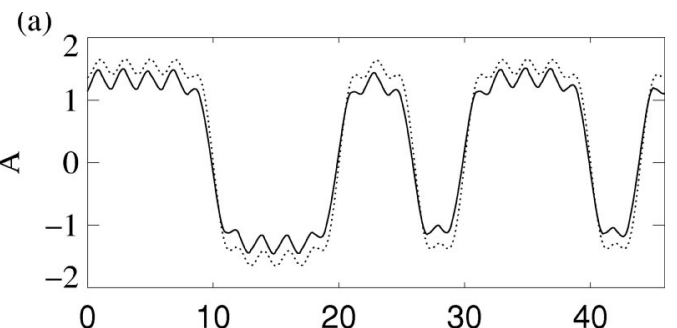

(b)

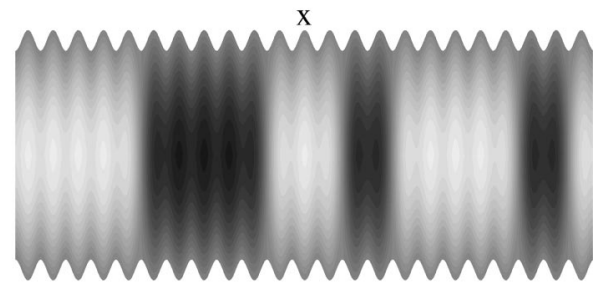

(c)

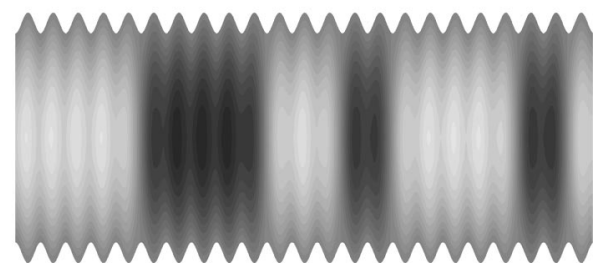

FIG. 3. Stationary solution of the STMA (3.1) compared to a fully two-dimensional simulation of Eq. (2.1) for the parameter values $d_{0}=d_{1}=0.1, \alpha=\pi, a=10, \phi=\pi$. (a) Stationary solution $A(x)$ of the STMA (dotted) and the on-axis values of the actual two-dimensional stationary solution $\psi(x, y=0)$ (solid). (b) The full two-dimensional solution of Eq. (2.1) represented on a gray-scale. White corresponds to the highest values of $\psi$ and black to the lowest. (c) Reconstruction of the two-dimensional field from the STMA $[\psi(x, y)=A(x) \sin (\pi \tilde{y})]$ on the same gray-scale as in (b).

\section{NUMERICAL STMA AND TWO-DIMENSIONAL TIME INTEGRATIONS}

In the previous section, we have shown both analytically and numerically that simple undulated boundaries may induce spatially chaotic steady solutions in our simple model (3.1). In this section, we discuss the accuracy and range of validity of the STMA and the physical relevance of its solutions by comparing with the numerical integration of the full model (2.1).

In the first place, we discuss the results of such integration for the particular case $\phi=\pi$ and $d_{0}=d_{1}=d$. Starting from random initial conditions the system, after a time long enough, settles in a disordered stationary configuration, as shown in Fig. 3. There we present the stationary configuration $A(x) \equiv A(x, t \rightarrow \infty)$ obtained by direct integration of Eq. (3.1). In the same plot, a longitudinal cross section of the asymptotic field obtained from a simulation with the full two-dimensional model is shown for comparison. We use as the initial condition of the two-dimensional problem the solution from the STMA for the same parameter values, $\psi$ $=A(x) \sin (\pi \tilde{y})$, to provide an approximate stationary solution to Eq. (2.1). After a short time of adjustment, the system settles in a stationary state that is very close to the initial approximation. The full two-dimensional field and its reconstruction from the STMA are also shown in Figs. 3(b) and 3(c), respectively. This figure reveals a strikingly accurate fit
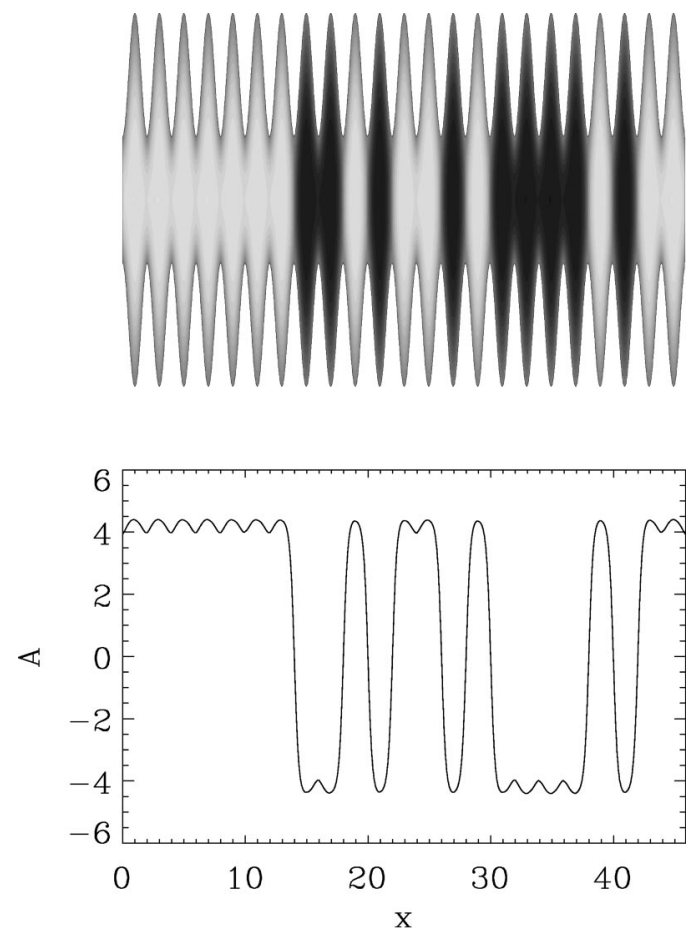

FIG. 4. The two-dimensional steady state obtained by simulation of the system (2.1) starting from random initial conditions. The amplitude of the field on the channel axis is shown in the lowest panel. The parameter values are $d=1.0, \alpha=\pi, a=20, \phi=\pi$.

of the STMA solution and the complete field simulation, a strong indication of the validity of the approximation as a tool for analysis. The maximum absolute error of the approximate solution is of the same order of magnitude in both the undulated channel and a rectangular-domain test case. This suggests that the error is mainly due to the truncation at the first linear transverse mode, but not to the peculiarities of the curved boundaries.

The accuracy of the STMA, $A(x, t) \sin (\pi \tilde{y})$, breaks down when the channel width increases or when strongly nonrectangular domains are considered. Nevertheless, we have also performed direct simulations of Eq. (2.1) for this last case. An example of the typical behavior is shown Fig. 4 for boundaries defined by $d_{0}=d_{1}=1.0, \alpha=\pi, a=20, \phi=\pi$. Notice that the resulting stationary configuration displays the same qualitative features of the STMA solutions: disordered distribution of kinks randomly pinned at some of the narrows of the channel. This is evidence of the fact that undulating boundaries may also be the source of stationary spatial chaos in a two-dimensional system (2.1) beyond the regime well described by a single transversal mode.

The results in Figs. 3 and 4 illustrate the physical mechanism behind the emergence of spatial chaos in our system. Let us remember that Eq. (2.1) evolves to minimize the potential (2.3). This minimization requires one to reduce as much as possible the length of the interfaces between $\psi_{+}$and $\psi_{-}$. Following this tendency, an interface that links the opposite lateral banks of the channel and is far from any other interface will evolve to lock into one of the narrows of the channel, where it is shorter than in any other position. Detaching the interface from the bank of the channel would imply a temporary increase of the potential $V$ due to the 
(a)

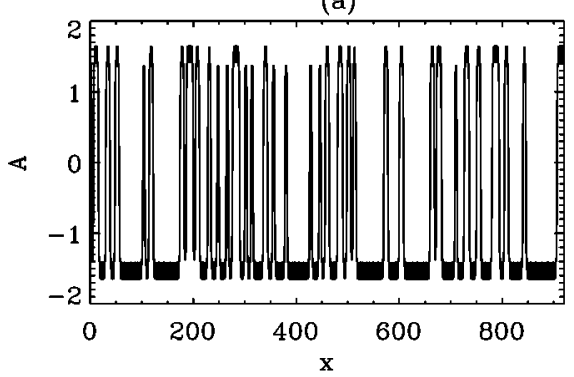

(c)

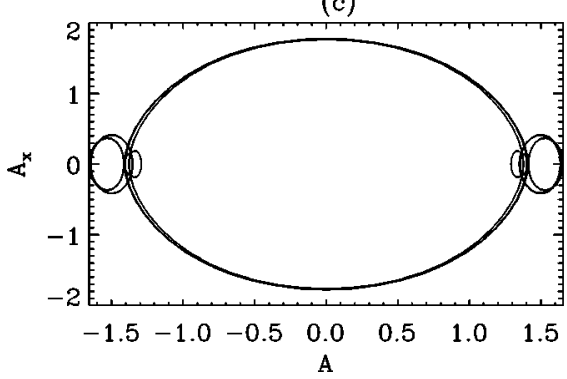

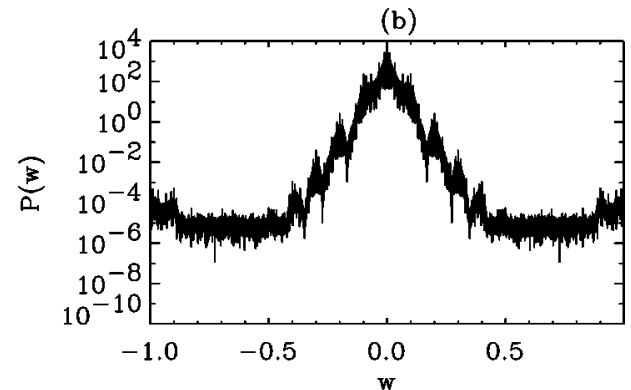

(d)

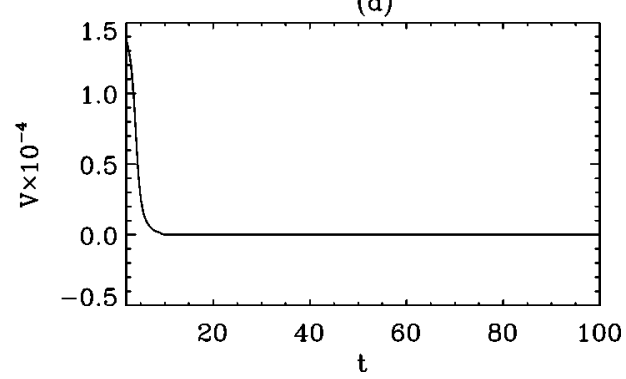

FIG. 5. One-dimensional solution of the system (3.1) showing a steady chaotic configuration, its spatial power spectrum, the trajectory in the projected phase space $\left(A, A_{x}\right)$, and the time evolution of the Lyapunov functional, showing that this is an attracting configuration. Parameter values as in Fig. 3, but for a longer system. necesary proliferation of new interfaces. Such a potential increase is not allowed by the dynamics. Hence, the random occupation (arising from random initial conditions) of the narrows of the channel by kinks and antikinks finally builds up a spatially chaotic stationary configuration. This argument, based on kink and interface dynamics, clearly applies beyond the range of validity of the STMA, where we have analytically established the existence of spatial chaos, as evidenced in Fig. 4. With this mechanism in mind, we can also conclude that not all the chaotic trajectories presented in Fig. 2 will lead to spatial chaos stable in time: only those corresponding to an energetically favorable (a local minima of the potential $V$ ) distribution of kinks will be reached under time evolution. In particular, the trajectory plotted in Fig. 2(b) corresponds to a temporally unstable configuration.

Let us now try to get further insight about the chaotic nature of the irregular spatial structures described above. Periodic boundary conditions in the longitudinal direction always force the system to converge not to a chaotic spatial configuration, but to a periodic one which we have shown may very well be of the maximal period. To justify the use of the chaotic qualifier we need to show that as the size of the system increases, these periodic configurations approach one that could be characterized as chaotic in some way. Of course, to numerically carry out this process we would need to consider very long channels. Unfortunately though, performing direct simulations on the fully two-dimensional model soon becomes computationally prohibitive as the number of the channel undulations increases. However, having demonstrated that the STMA accurately describes the qualitative features of the full model, we can concentrate our attention on the behavior of the approximate model Eq. (3.1).

In Fig. 5 we summarize the results from the numerical integration of Eq. (3.1) using the same parameter values as in Fig. 3 with the exception of the domain size which now is much larger. An asymptotically stable configuration is shown in (a), while (c) displays the projection of the trajectory in phase space $\left(A, A_{x}\right)$. The power spectrum of $A(x)$ plotted in Fig. 5(b) shows the typical broadband feature characteristic of chaotic trajectories. As a further indication of the (approximately) chaotic nature of this configuration, we have shown in Fig. 6 the stroboscopic map constructed from the trajectory in Fig. 5, taking phase-space points at times integer multiples of $T$. This map reveals an incipient self-similar fractal structure, also common in chaotic trajectories. All these facts together give compelling evidence that the trajectory, although $L$ periodic by construction, develops chaotic features as the system size increases.

Finally, as an illustrative measure of the asymptotic stability of this solution, Fig. 5(d) displays the value of the Lyapunov potential $V[\psi]$ evaluated along the time evolution of the field $\widetilde{\psi}(x, \tilde{y}, t)=A(x, t) \sin (\pi \tilde{y})$. The functional decreases in time, confirming the consistency of the STMA with the exact dynamics of Eq. (2.2), and the potential asymptotically approaches a constant value indicating that the field has reached a local minimum of $V$.

\section{CONCLUSIONS}

We have given evidence of the existence of stationary, stable, longitudinally chaotic spatial configurations induced by undulated boundaries in a simple two-dimensional reaction-diffusion model that does not otherwise display any kind of chaos. We have demonstrated that these type of boundaries can be convincingly mapped into spatially periodic parametric modulations in a one-dimensional approximation to the original system. In a dynamical-systems approach to the study of stationary solutions, these modulations play the role of a temporal time-periodic forcing capable to drive a nonlinear second-order ODE (ordinary differential equation) into chaotic behavior. The diffusive character of our original model ensures precisely that the relevant ODE is in fact, second order and that the presence of chaotic stationary solutions is expected at this level of approximation. Previous results exist $[6,20,21]$ showing that spatial periodic modulation of some parameters intrinsic to the dynamics may originate spatial chaos in relatively simple reaction diffusion models. However, to our knowledge, the present is the first example in which a straightforward dynamical systems approach is used to establish the existence of disorder in 

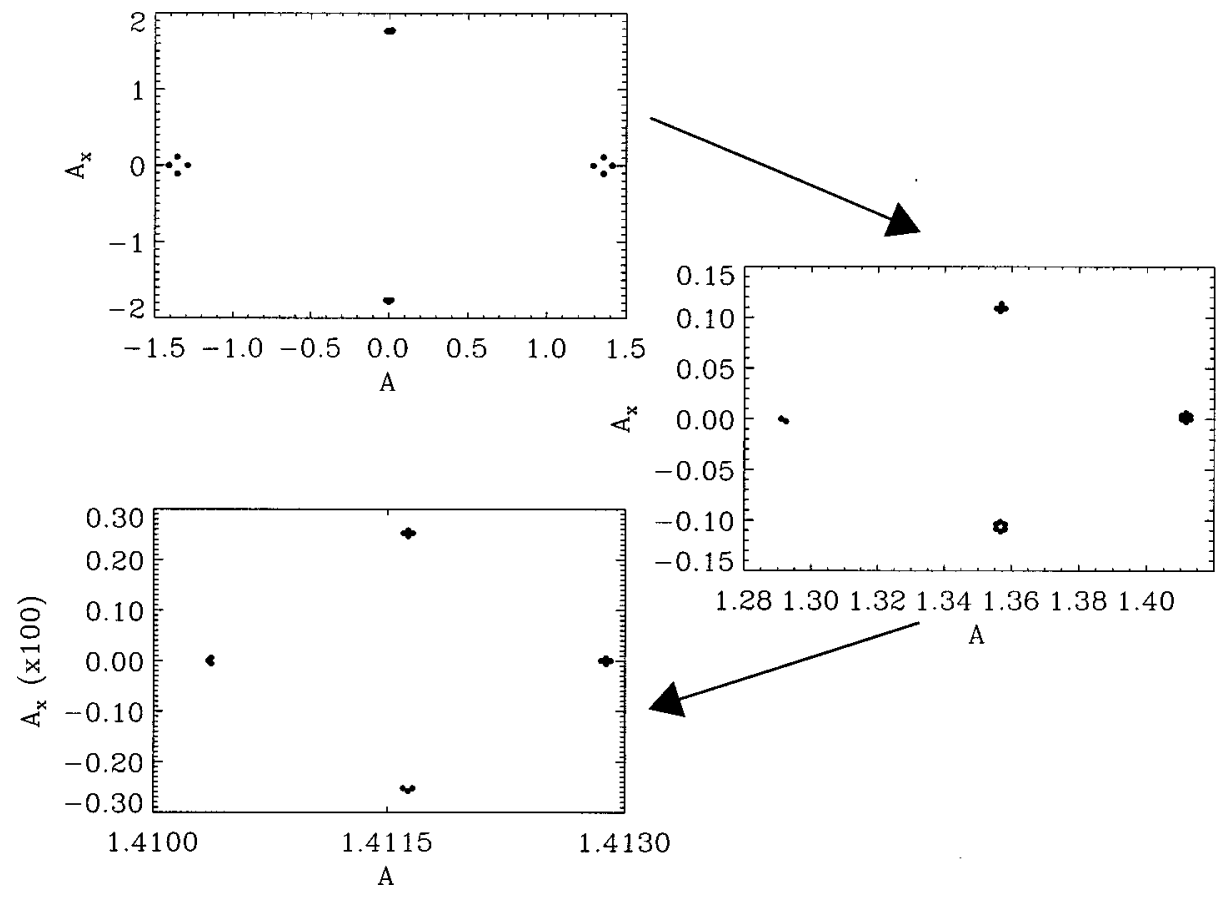

FIG. 6. Stroboscopic map of the trajectory shown in Fig. 5. Two succesive amplifications are shown, displaying a self-similar structure typical of a fractal. two-dimensional systems due to the influence of the boundaries.

The consequence of our analysis is that chaotic configurations should exist in virtually any of the experimental systems commonly used to study pattern formation, provided that boundary conditions such as those studied here are imposed. The stability of these chaotic configurations should be discussed in each particular case. While in our model stability comes from the tendency of the dynamics to minimize Eq. (2.3), therefore minimizing interface lengths and leading to pinning of these interfaces to the narrows of the channel, the mechanism for stability in other systems may be different. Apart from the direct application to pattern forming systems, the idea of boundary-generated spatial chaos could be speculatively transferred to other nonlinear extended dy- namical systems of interest. For example, it is possible that low Reynolds number fluid flows through a spaceperiodically perturbed pipeline, or even through a realistic channel of shape similar to the ones considered here, can display frozen spatial chaos [17]. A numerical search for this manifestation of "frozen turbulence" at the level of NavierStokes equations is currently in progress. We expect this observation to promote also experimental work both in the area of pattern formation and in hydrodynamics.

\section{ACKNOWLEDGMENTS}

Financial support from PB94-1167 and PB97-0141C02-01 is greatly acknowledged.
[1] H. Bai-Lin, Chaos (World Scientific, Singapore, 1990).

[2] E. Ott, Chaos in Dynamical Systems (Cambridge University Press, Cambridge, England, 1993).

[3] K. Alligood, T. Sauer, and J.A. Yorke, Chaos: an Introduction to Dynamical Systems (Springer-Verlag, New York, 1997).

[4] M.C. Cross and P.C. Hohenberg, Science 263, 1569 (1994).

[5] M.C. Cross and P.C. Hohenberg, Rev. Mod. Phys. 65, 851 (1993).

[6] P. Coullet, C. Elphick, and D. Repaux, Phys. Rev. Lett. 58, 431 (1987).

[7] M.I. Rabinovich, A.L. Fabrikant, and L.Sh. Tsimring, Usp. Fiz. Nauk 162, 1 (1992) [Sov. Phys. Usp. 35, 629 (1992)].

[8] R. Montagne, E. Hernández-García, and M. San Miguel, Phys. Rev. Lett. 77, 267 (1996); e-print chao-dyn/9604004; R. Montagne, E. Hernández-García, A. Amengual, and M. San Miguel, Phys. Rev. E 56, 151 (1997); e-print chao-dyn/9701023.

[9] H-C. Chang, Annu. Rev. Fluid Mech. 26, 103 (1994).

[10] C. Elphick, E. Meron, and E.A. Spiegel, Phys. Rev. Lett. 61,
496 (1988); C. Elphick, E. Meron, and E.A. Spiegel, SIAM (Soc. Ind. Appl. Math.) J. Appl. Math. 50, 490 (1990); C. Elphick, E. Meron, J. Rinzel, and E.A. Spiegel, J. Theor. Biol. 146, 249 (1990).

[11] N.J. Balmforth, Annu. Rev. Fluid Mech. 27, 335 (1996).

[12] D. Ruelle, Physica D 7, 40 (1983).

[13] A.C. Newell and Y. Pomeau, J. Phys. A 26, L429 (1993).

[14] P. Bak, Rep. Prog. Phys. 45, 587 (1982).

[15] M. Lowe, J.P. Gollub, and T.C. Lubensky, Phys. Rev. Lett. 51, 786 (1983); M. Lowe, B.S. Albert, and J.P. Gollub, J. Fluid Mech. 173, 253 (1986); P. Coullet, Phys. Rev. Lett. 56, 724 (1986).

[16] S.W. Jones, O.M. Thomas, and H. Aref, J. Fluid Mech. 209, 335 (1989).

[17] Y. Le Guer, C. Castelain, and H. Peerhossaini (unpublished).

[18] J.H.E. Cartwright, M. Feingold, and O. Piro, in Mixing: Chaos, and Turbulence, edited by H. Chaté and E. Villermaux (Kluwer, Dordrecht, 1999).

[19] E. Hernández-García, M. San Miguel, R. Toral, and J. Viñals, 
Physica D 61, 159 (1992).

[20] K.A. Gorskov, L.N. Korzinov, M.I. Rabinovich, and L.S. Tsimring, J. Stat. Phys. 74, 1033 (1994).

[21] M.A. Malkov, Physica D 95, 62 (1996).

[22] V.S. Afraimovich, A.B. Ezersky, M.I. Rabinovich, M.A. Shereshevsky, and A.L. Zheleznyak, Physica D 58, 331 (1992).

[23] J.D. Gunton, M. San Miguel, and P.S. Sahni, The Dynamics of First Order Phase Transitions, in Phase Transitions and Critical Phenomena Vol. 8, edited by C. Domb and J.L. Lebowitz (Academic, New York, 1983).

[24] J.D. Murray, Mathematical Biology (Springer, Berlin, 1993).

[25] R. Montagne, E. Hernández-García, and M. San Miguel, Physica D 96, 47 (1996); e-print cond-mat/9508115; M. San
Miguel, R. Montagne, A. Amengual, and E. HernándezGarcía, in Instabilities and Nonequilibrium Structures $V$, edited by E. Tirapegui and W. Zeller (Kluwer, Dordrecht, 1996); e-print patt-sol/9502003.

[26] P. Collet, Nonlinearity 7, 1175 (1994); e-print patt-nol/ 9310002.

[27] For similar multiple scales analysis applied to fluid flow in a varying curved channel, see, e.g., A. Doelman, W. Eckhaus, R. Kuske, and R. Schilen, in Nonlinear Dynamics and Pattern Formation in the Natural Environment, edited by A. Doelman and A. van Harten (Longmans, New York, 1995).

[28] A.H. Nayfeh and B. Balachandran, Applied Nonlinear Dynamics (Wiley-Interscience, New York, 1995). 